\title{
NOVEDADES NOMENCLATURALES EN EL VOL. 66 (1) DE GRAELLSIA
}

Arachnida, Acari, Lethaxonidae

Transitia n. gen.

Transitia carlosi n. sp.

Arachnida, Acari, Ctenobelbidae

Bifurcobelba $\mathrm{n}$. subg.

Caucasiobelba n. subg.

Ctenobelba (Bifurcobelba) iberica $\mathrm{n} . \mathrm{sp}$.

Ctenobelba (Caucasiobelba) reticulata n. sp.
Coleoptera, Meloidae

Meloe (Eurymeloe) marianii Kaszab, 1938 n. syn. de Meloe (Eurymeloe) saharensis Chobaut, 1898

Meloe otini Peyerimhoff, 1949 n. syn. de Meloe (Eurymeloe) saharensis Chobaut, 1898

Diptera, Chironomidae

Trissopelopia bifurcata $\mathrm{n}$. sp. 